University of Wollongong

Research Online

Faculty of Informatics - Papers (Archive)

Faculty of Engineering and Information

Sciences

2008

\title{
Adaptive subband selection in OFDM-based cognitive radios for better system coexistence
}

Pingzhou Tu

University of Wollongong, pt015@uow.edu.au

Xiaojing Huang

University of Wollongong, huang@uow.edu.au

Eryk Dutkiewicz

University of Wollongong, eryk@uow.edu.au

Follow this and additional works at: https://ro.uow.edu.au/infopapers

Part of the Physical Sciences and Mathematics Commons

\section{Recommended Citation}

Tu, Pingzhou; Huang, Xiaojing; and Dutkiewicz, Eryk: Adaptive subband selection in OFDM-based cognitive radios for better system coexistence 2008.

https://ro.uow.edu.au/infopapers/3222

Research Online is the open access institutional repository for the University of Wollongong. For further information contact the UOW Library: research-pubs@uow.edu.au 


\title{
Adaptive subband selection in OFDM-based cognitive radios for better system coexistence
}

\author{
Abstract \\ In an environment of shared radio spectrum, multiple systems may interfere with each other and cause \\ significant impacts on system coexistence. In this paper we propose an adaptive subband selection \\ technique based on orthogonal frequency division multiplexing (OFDM) to avoid interference for better \\ system coexistence when multiple systems are operating in the same unlicensed industrial, scientific and \\ medical (ISM) bands. Under the assumption that the interference power level and the interfered frequency \\ bands are identified at the receiver, interference thresholds, determined over both Gaussian and multipath \\ fading channels, are applied to adaptively select the transmission subbands so that interference is \\ avoided and the system coexistence issues are relaxed. To verify the effectiveness of the proposed \\ adaptive subband selection method, the system bit error rates (BERs) under different interference levels \\ are simulated and compared.

\section{Disciplines} \\ Physical Sciences and Mathematics

\section{Publication Details} \\ P. Tu, X. Huang \& E. Dutkiewicz, "Adaptive subband selection in OFDM-based cognitive radios for better \\ system coexistence," in 3rd International Conference on Cognitive Radio Oriented Wireless Networks and \\ Communications, 2008, pp. 1-6.
}




\title{
Adaptive Subband Selection in OFDM-Based Cognitive Radios for Better System Coexistence
}

\author{
Pingzhou Tu, Xiaojing Huang, Eryk Dutkiewicz \\ Email: \{pt015, huang, eryk\}@uow.edu.au \\ School of Electrical, Computer and Telecommunications Engineering \\ University of Wollongong, Australia
}

\begin{abstract}
In an environment of shared radio spectrum, multiple systems may interfere with each other and cause significant impacts on system coexistence. In this paper we propose an adaptive subband selection technique based on orthogonal frequency division multiplexing (OFDM) to avoid interference for better system coexistence when multiple systems are operating in the same unlicensed industrial, scientific and medical (ISM) bands. Under the assumption that the interference power level and the interfered frequency bands are identified at the receiver, interference thresholds, determined over both Gaussian and multipath fading channels, are applied to adaptively select the transmission subbands so that interference is avoided and the system coexistence issues are relaxed. To verify the effectiveness of the proposed adaptive subband selection method, the system bit error rates (BERs) under different interference levels are simulated and compared.
\end{abstract}

\section{INTRODUCTION}

Coexistence is the ability of two or more nodes or entities to share a common frequency band [1]. One of the main factors to limit the coexistence between systems sharing a common frequency band is the interference from all sources.

To avoid interference between systems, many techniques have been proposed. Generally, these techniques can be classified into two categories. One is the collaborative mechanism for wireless systems capable of sharing detailed information in real-time about their predefined occupancy of time, frequency, space and power, in which the coexistence is realized by dynamically managing transmission frequency, controlling emission power and rescheduling in time. The other is the noncollaborative one, in which one or more systems must sense the environment and take a corresponding action [2]. With this method radios scan the channels in a service band and select the channel with the lowest received signal strength indicator (RSSI) for data transmission [3]. Since the selected signal band with the lowest RSSI may have interference above an acceptable threshold, this interference will lead to a large error probability. For those methods of managing frequency, controlling power and time, the most advanced systems are approaching Shannon's channel capacity limit. Further increase in capacity would require additional system bandwidth [4].

Cognitive radio $(\mathrm{CR})$, as one of the noncollaborative techniques for improving coexistence, can sense a wireless radio scene and identify interference. It aims at promoting the efficiency of the spectrum utilization and the coexistence between current users and primary users. Based on CR and orthogonal frequency division multiplexing (OFDM) techniques, one attempt to avoid interference is to exclude some sub-bands experiencing deep-fading by sculpting the corresponding subcarrier group [5]. However, the reallocation of the transmission signal power for the excluded subbands to the remaining sub-bands increases the power of remaining subbands and introduces interferences to the primary users. Moreover, it requires more advanced techniques to suppress the sidelobes from rectangular window [6], which is sometimes known as a Dirichlet window.

In this paper we propose an adaptive subband selection technique to avoid interference for the coexistence in an OFDM-based system operating in unlicensed ISM bands. Under the assumption that the interference power level and frequency bands can be identified based on cognitive radio (CR) techniques, the subbands of the transmitted signal are adaptively selected so that interference is avoided and the system coexistence is improved. The proposed technique provides a greatly simplified solution to the system coexistence issue.

The remainder of this paper is organized as follow. In Section II the system model is introduced. In Section III, the methods of multiple subband signal generation and adaptive subband selection are described. In Section IV we determine the interference thresholds for systems over Gaussian and multipath fading channels using analytical and numerical means respectively. In Section V, the system bit error rates (BERs) in different channel conditions are simulated and discussed. Finally, we draw our conclusions in Section VI.

\section{SYSTEM MODEL}

The system is an OFDM-based cognitive radio (CR) and consists of a CR transmitter and a $\mathrm{CR}$ receiver and channels. The CR transmitter is composed of a spread spectrum modulator and a filter. The modulator spreads the baseband signal into a signal with multiple subbands. Each subband contains the whole transmitted information. The generation of the multiple subband signal will be investigated in the next section. The receiver consists of a radio scene sensor, an adaptive filter and a demodulator. The function of the radio scene sensor is to scan the spectrum, detect interference power level, and identify interfered signal subbands. After the interference signal power level and the interfered subbands 
are identified, the receiver can adaptively select transmission subbands in terms of predetermined interference thresholds.

When signals propagate in fading multipath channels, the presence of reflecting objects and scatterers may result in signal fading. Let $h(m)$ be the impulse response of the multipath channel, and $y(m)$ the transmitted signal passing through the fading channel, the received equivalent low-pass signal $r(m)$ is viewed as the convolution between $h(m)$ and $y(m)$ plus noise $v(m)$ and interference signal $j(m)$, i.e.,

$$
r(m)=h(m) \otimes y(m)+v(m)+j(m),
$$

where $v(m)$ represents the complex-valued white Gaussian noise corrupting the signal, $j(m)$ represents the interference signal. In the frequency domain (1) is equivalent to the output of a fast Fourier Transform (FFT) demodulator, i.e.,

$$
R(k)=H(k) \cdot Y(k)+V(k)+J(k),
$$

where $R(k), Y(k), H(k), V(k)$ and $J(k)$ denote the frequency representations of the received signal, transmitted signal, channel impulse response, noise and interference signal $r(m), y(m), h(m), v(m)$ and $j(m)$ respectively.

In the following discussion we assume that the interference signal types, interference power levels and frequency bands have been detected by the CR system.

\section{MULTIPLE SUBBAND SIGNAL}

\section{A. Generation of the Multiple Subband Signal}

To enable adaptive subband selection, the first challenge is how to generate a signal that can adaptively change the occupied bandwidth without losing transmitted information.

Fig.1 indicates the generation process of such a multiple subband signal. Let $a_{i}(i=0,1, \cdots, N)$ denotes the $i^{\text {th }}$ complex-valued symbol of the $N$ quadrature phase shift keying (QPSK) symbols to be transmitted. The $N$ QPSK symbols are modulated by the modulator, and then interleaved by the interleaver to form a multiple subband OFDM signal. After serial to parallel conversion the $N \times 1$ parallel QPSK symbols modulate the corresponding $N$ subcarriers. If the OFDM symbol period is $T_{s}, f_{i}=\frac{i}{T_{s}}$ denotes the $i^{t h}$ subcarrier frequency of the $N$ orthogonal subcarriers, and $a_{i}$ modulates the $i^{\text {th }}$ subcarrier at time $t=\frac{n}{N} T_{s}, n=0,1, \cdots, N-1$, where $n$ is the sampling time index. The modulated symbol on the $i^{t h}$ subcarrier and the $n^{t h}$ time instant is written as

$$
y_{i}(n)=a_{i} e^{j 2 \pi f_{i} t}=a_{i} e^{j 2 \pi n i / N} .
$$

In the OFDM symbol duration $T_{s}$, each element of the $N \times 1$ data symbol vector modulates the same subcarrier $N$ times, so that $N$ elements in the vector generate an $N \times N$ sample matrix after modulation. In consequence, $N$ replicas of each data symbol are produced in symbol duration $T_{s}$ by the modulator as displayed in Fig.1.

After the modulation, the $N \times N$ samples are shifted in time and placed on different time slots to form a serial sequence of length $N^{2}$ in the time domain. This operation is a process of interleaving. The interleaving can be realized by shifting $N$ modulated subcarriers on different time slots and adding them together. For instance, the $i^{t h}$ subcarrier of the $N$ subcarriers is shift by $i \cdot \tau$, where $\tau$ is the time interval, $\tau=\frac{T_{s}}{N^{2}}$, then, the $N$ shifted subcarriers are added together to form one OFDM symbol with $N^{2}$ samples. The $m^{t h}$ sample in the OFDM symbol, denoted by $y(m), m=n N+i=0,1, \cdots, N^{2}-1$, can be mathematically written as

$$
y(m)=\sum_{i=1}^{N} \sum_{n}^{N} y_{i}(n) \delta[m-i-n N]
$$

where $\delta[m-n N-i]=\left\{\begin{array}{l}1, \text { for } m=n N+i \\ 0, \text { for } m \neq n N+i .\end{array}\right.$ is the Dirac impulse. It can be simplified as

$$
y(m)=y(n N+i)=y_{i}(n)
$$

where $m=n N+i$ and $n, i=0,1, \cdots, N-1$.

The mathematical equations above can be interpreted as follows. All $N$ samples in a column of the $N \times N$ matrix are taken out, shifted in time, and then placed in different time slots, instead of being superimposed together as in conventional OFDM systems.

The frequency domain representation of the multiple subband OFDM signal can be obtained by performing a fast Fourier Transform (FFT) operation on the signal $y(m)$ in time domain, i.e,

$Y(k)=F F T(y(m))=N \sum_{i=0}^{N-1} a_{i} e^{-j \frac{2 \pi}{N^{2}} k i} \underbrace{\frac{1}{N} \sum_{n=0}^{N-1} e^{-j \frac{2 \pi}{N}(k-i) n}}_{\delta\left((k-i)_{N}\right)}$

or $Y(p N+i)=Y_{p}(i)=N a_{i} e^{-j \frac{2 \pi}{N^{2}}(p N+i) i}, \quad k=p N+i$, where $i, p=0,1, \cdots, N-1$.

Equation above indicates that the OFDM symbol consists of $N$ subbands. Each data symbol $a_{i}$ is modulated on the $i^{\text {th }}$ subcarrier of each subband, and the signal spectrum is spread $N$ times. Fig. 2 indicates the signal spectrum when $N=4$. It is seen from Fig.2 that the multiple subband OFDM symbol contains 4 subbands and the modulated data symbol on the $i^{\text {th }}(i=1,2,3,4)$ subcarrier appears in all the 4 subbands. The signal spectrum is expanded to 4 subbands, each of which contains 4 orthogonal subcarriers modulated by the same transmitted data symbols. Due to the channel frequency-selective fading, these power spectrum densitiesof the subbands have different attenuations.

\section{B. Interference Avoidance}

When the multiple subband OFDM signal is passing through a multipath fading channel, since the fading channel dissipates the signal energy, the received signals are faded in different subbands. Meanwhile, an interference signal will be imposed on the transmitted signals. To avoid interference at the receiver so that the system performance can be improved, the interfered subbands should be removed from the received signal once the interference power lever is above a predetermined threshold. The mechanism is explained in more detail below. 
Assume the value of the threshold is $\gamma_{0}$. The derivation method of the threshold will be discussed in the next section. If the interference superimposed on a subband is greater than the threshold value $\gamma_{0}$, then the interference would cause severe adverse effects on the desired signal. In order to achieve better system BER performance, the interfered subband should be excluded. If the interference is lower than the threshold value $\gamma_{0}$, the interference can be tolerated and the interfered subbands are kept in the transmission bands. Otherwise, if we remove the interfered subbands with interference lower than the threshold, the system performance will be worse than that of the system with the interfered subbands, since when we remove the interfered subbands, the signal energy is removed at the same time.

Fig. 3 interprets the fundamental principle of interference avoidance, in which a 4 subband OFDM signal in the unlicensed $2.4 \mathrm{GHz}$ ISM frequency band is faded and interfered, but only two of the subbands with interference over the threshold are removed. Fig.3a displays the OFDM signal of 4 subbands interfered with different power levels. As displayed in Fig. $3 b$ the subbands with interference over the interference threshold $\gamma_{0}$ is sculpted by using an adaptive filter. The other two interfered subbands remain in the transmission bands since their interference levels are lower than the threshold.

\section{DETERMINATION OF THRESHOLDS}

\section{A. Thresholds over Gaussian Channels}

Assume that $M$ of the $N$ subbands are received and used for demodulation, each of which has signal power $P_{S}$. If the noise power spectral density is $N_{0}$, and the total interference signal power in the observed frequency band is $P_{J}$ with bandwidth $B_{J}$, which is distributed in $l$ subbands of the transmitted $M$ subband OFDM signal. Then, the signal power of the $M$ subbands is $M \cdot P_{S}$, and the signal-to-interference noise ratio $(S I N R)$ is expressed as

$$
S I N R_{M}^{(1)}=\frac{M \cdot P_{S}}{N_{0} M \cdot B+P_{J}}=\frac{S N R}{1+I N R},
$$

where $S N R=\frac{M P_{S}}{M N_{0} B}, I N R=\frac{P_{J}}{N_{0} M B}$ is interference noise ratio, $B$ is the bandwidth of each subband.

Let $G_{p}$ denote the processing gain for each subband, the total SINR, after considering the processing gain is the sum of M subbands $S I N R_{M}^{(1)}$, denoted by

$$
S I N R^{(1)}=M \cdot G_{p} \cdot S I N R_{M}^{(1)}=\frac{M \cdot G_{p} \cdot S N R}{1+I N R} .
$$

Similarly, if the interfered subbands are cut off, the interference energy $P_{J}$ is correspondingly completely removed. Thus, the total signal-to-interference noise ratio (SINR), after the interfered subbands are removed, can be expressed as

$$
S I N R_{M-l}^{(2)}=\frac{(M-l) \cdot P_{S}}{N_{0}(M-l) \cdot B}=S N R .
$$

Similarly, the total SINR, after the $l$ interfered subbands are removed and considering the processing gain, is the sum of

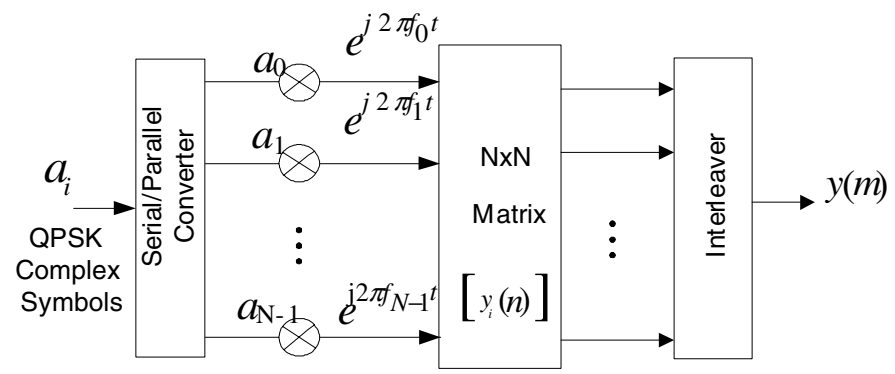

Fig. 1. Multiple subband signal modulation.

$M-l$ subbands $S I N R_{M-l}^{(2)}$ and denoted by

$S I N R^{(2)}=(M-l) \cdot G_{p} \cdot S I N R_{M-l}^{(2)}=(M-l) \cdot G_{p} \cdot S N R$.

Since the signal power is distributed in the whole bands, after the $l$ interfered subbands are removed, the signal energy distributed in the $l$ subbands is also removed, which causes the loss of some portions of the signal power. Thus, it is essential to decide how many of the $l$ subbands should be removed for interference suppression. This compromise can be realized in terms of the adaptively computed interference threshold. In Gaussian channels, the BER performance is determined by the normalized signal-to-noise ratio (SNR) or signal-tointerference noise ratio (SINR). System performance with higher SINR will be better. Thus, the interference thresholds are obtained by letting the $S I N R^{(2)}$ greater than $S I N R^{(1)}$, i.e,

$$
S I N R^{(2)}>S I N R^{(1)}
$$

Substituting $S I N R^{(2)}$ in (8) and $S I N R^{(1)}$ in (6) into (9), we have the following expression

$$
I N R>\frac{l}{M-l}=\gamma_{0},
$$

where $\gamma_{0}$ is the interference threshold.

It can be seen from (10) that the threshold $\gamma_{0}$ of the $I N R$ is related to the number of transmitted subbands $M$ and the number of interfered subbands $l$ over Gaussian channels. Given the interfered subband number $l(l=1,2,4,8)$, the $I N R$ threshold decreases with the increase of the number $M$ of the transmitted subbands. If the number $M$ of the transmitted subbands is given, the $I N R$ threshold increases with the increase of the interfered subband number $l$.

\section{B. Thresholds over Multipath Fading Channels}

In a multipath fading channel, since the channel fading is random, we cannot obtain the same thresholds as those in Gaussian channel. However, it is possible for us to get an estimation of the thresholds by statistically analyzing system BER performance over multipath fading channels.

Recalling (6) and considering the multipath fading effects, system BER performance before the interfered subbands are 
Table 1: Threshold $\gamma_{0}$ Comparison between Gaussian channels and Multipath Channels.

\begin{tabular}{|c|c|c|c|c|c|c|c|c|c|c|}
\hline \multirow{2}{*}{$\begin{array}{l}\text { Interfered } \\
\text { Subbands }\end{array}$} & \multicolumn{2}{|c|}{ Thresholds (dB) $M=2$} & \multicolumn{2}{|c|}{ Thresholds (dB) $\mathrm{M}=4$} & \multicolumn{2}{|c|}{ Thresholds (dB) $M=8$} & \multicolumn{2}{|c|}{ Thresholds (dB) $M=16$} & \multicolumn{2}{|c|}{ Thresholds $(\mathrm{dB}) \mathrm{M}=32$} \\
\hline & Gauss. & Multi. & Gauss. & Multi. & Gauss. & Multi. & Gauss. & Multi & Gauss. & Multi. \\
\hline $1=1$ & 0 & 4.9 & -4.8 & -1.7 & -8.5 & -5.5 & -11.8 & -11.0 & -14.9 & -14.5 \\
\hline $1=2$ & - & - & 0 & 3.3 & -4.8 & -2.0 & -8.5 & -7.5 & -11.8 & -11.5 \\
\hline $1=4$ & - & - & - & - & 0 & 3.0 & -4.8 & -4.0 & -8.5 & -8.5 \\
\hline $1=8$ & $\overline{-}$ & - & $\overline{-}$ & - & - & - & 0 & 1.0 & -4.8 & -4.5 \\
\hline Discrepancy & \multicolumn{2}{|c|}{$\approx 4.9$} & \multicolumn{2}{|c|}{$\approx 3.2$} & \multicolumn{2}{|c|}{$\approx 3$} & \multicolumn{2}{|c|}{$\approx 1.0$} & \multicolumn{2}{|c|}{$\approx 0.3$} \\
\hline
\end{tabular}

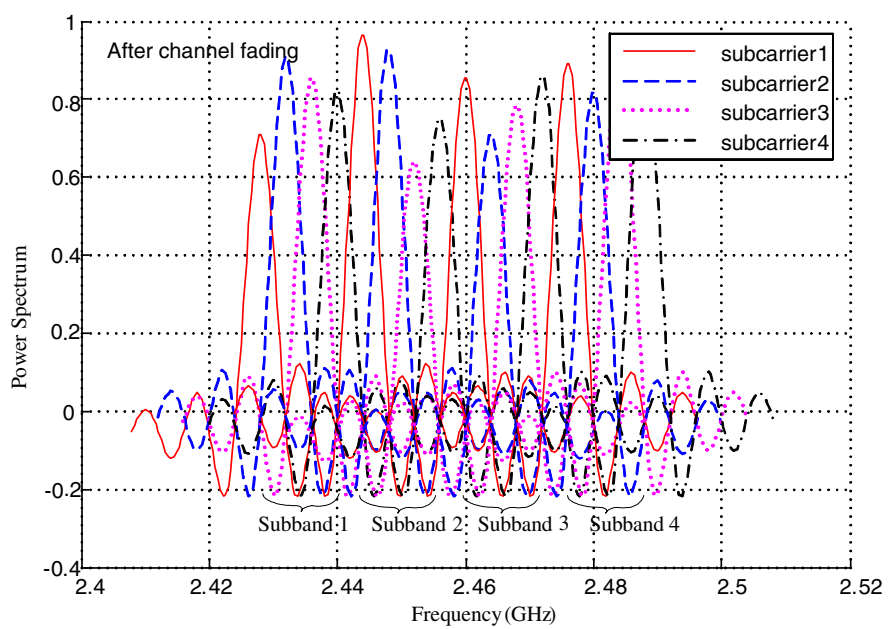

Fig. 2. Channel fading effects on subbands when subcarrier number $\mathrm{N}=4$.
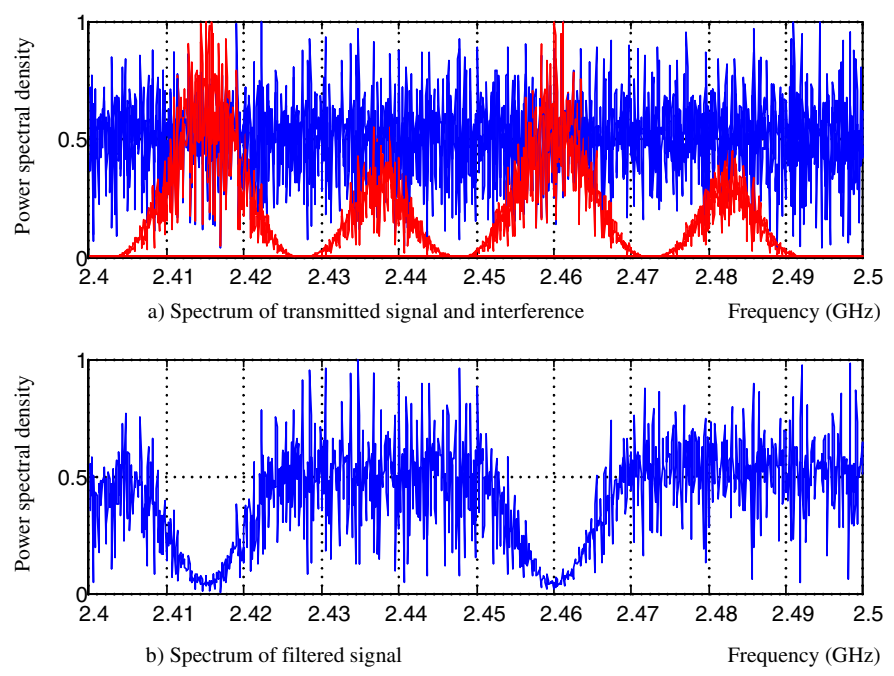

Fig. 3. Subband selection by using adaptive filter.

removed can be expressed by using Q function as

$$
\begin{gathered}
P_{e}^{(1)}=E_{C}\left[Q\left(\sqrt{\sum_{p=0}^{M-1}\left|H_{p}\right|^{2} S I N R^{(1)}}\right)\right] \\
=E_{C}\left[Q\left(\sqrt{\sum_{p=0}^{M-1}\left|H_{p}\right|^{2} \frac{S N R}{1+I N R}}\right)\right],
\end{gathered}
$$

where $E_{C}(\cdot)$ denotes the statistical expectation of the con-

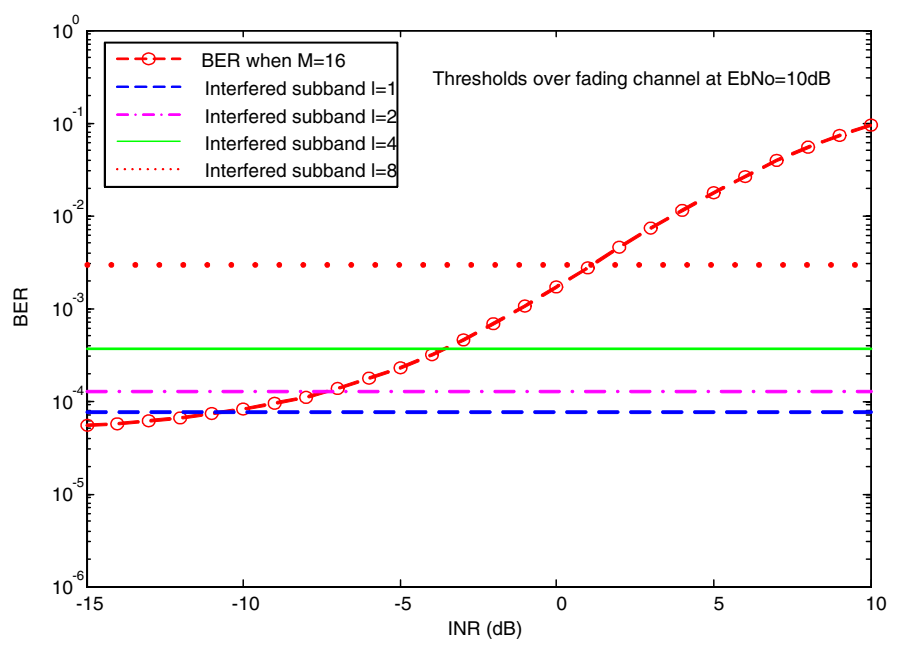

Fig. 4. INR thresholds over multipath fading channel.

ditional error probability over multipath fading channels, $\sum_{p=0}^{M-1}\left|H_{p}\right|^{2}$ is the sum of the $M$ multipath fading channel coefficients on the $M$ subcarriers. Due to the QPSK modulation, $S N R=\frac{2}{M} E_{b} / N_{0}$ where $E_{b} / N_{0}$ is the signal bit energy to noise spectrum density ratio [7]. The (11) can be rewritten as

$$
P_{e}^{(1)}=E_{C}\left[Q\left(\sqrt{\frac{1}{M} \sum_{p=0}^{M-1}\left|H_{p}\right|^{2} \frac{2 \cdot E_{b} / N_{0}}{1+I N R}}\right)\right]
$$

Similarly, recalling (8), the BER performance of the system over multipath fading channels, after the interfered subbands are removed, can be derived as

$$
P_{e}^{(2)}=E_{C}\left[Q\left(\sqrt{\sum_{p=0}^{M-l-1}\left|H_{p}\right|^{2} \frac{2 \cdot E_{b} / N_{0}}{M}}\right)\right],
$$

where $E_{C}(\cdot), H_{p}, E_{b} / N_{0}$ have the same meanings as defined in (11) and (12).

According to (12), we simulate the system BER performance over multipath fading channels with the increase of the INR when the transmission subband number is 16 and $E_{b} / N_{0}=10 \mathrm{~dB}$, which is displayed in Fig.4. After the interfered subbands (the number of the subbands is $l=1,2,4,8$ respectively) are removed, according to (13) and with the same $E_{b} / N_{0}$, the BER performance of the system over multipath fading channels are shown as flat lines in Fig.4. The cross points are the interference thresholds $\gamma_{0}$ corresponding to $l=1$, 2,4 and $l=8$. It can be seen from Fig. 4 that the interference 


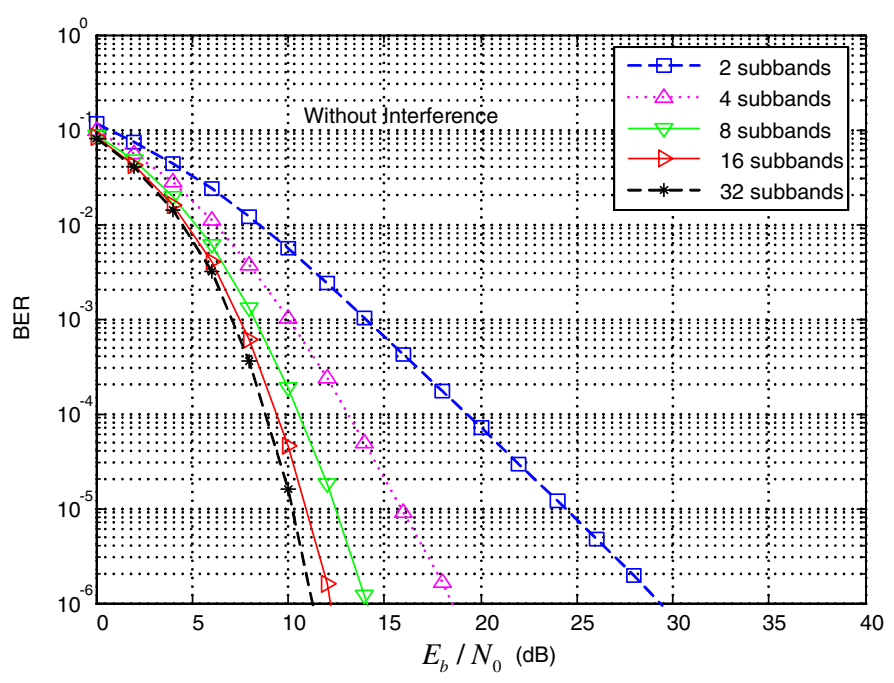

Fig. 5. BER performance without interferences in fading channel.

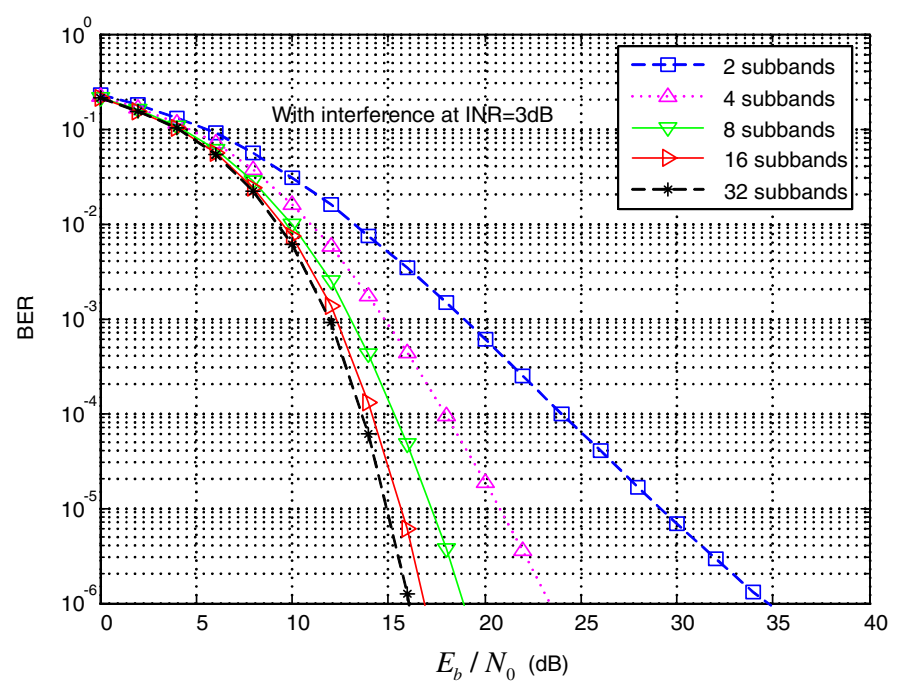

Fig. 6. BER performance with interferences in fading channel.

thresholds are $-11 \mathrm{~dB},-7.5 \mathrm{~dB},-4.0 \mathrm{~dB}$ and $1 \mathrm{~dB}$. The corresponding thresholds at the same conditions over a Gaussian channel can be calculated from (10). Similarly, the interference thresholds in a multipath fading channel when transmission subbands $M=2,4,8,16$ and 32 can be also determined. The thresholds over both multipath fading channels and Gaussian channels are presented in Table 1. It is observed from Table 1 that the threshold discrepancies between a multipath fading channels and a corresponding Gaussian channel decrease with the increase of the transmission subband number and the discrepancy is approximated zero when the transmission subband number $M=32$. That is to say, we can use the threshold over a Gaussian channel to replace the threshold over the corresponding multipath fading channel if the number of transmitted subbands is greater than 32 .

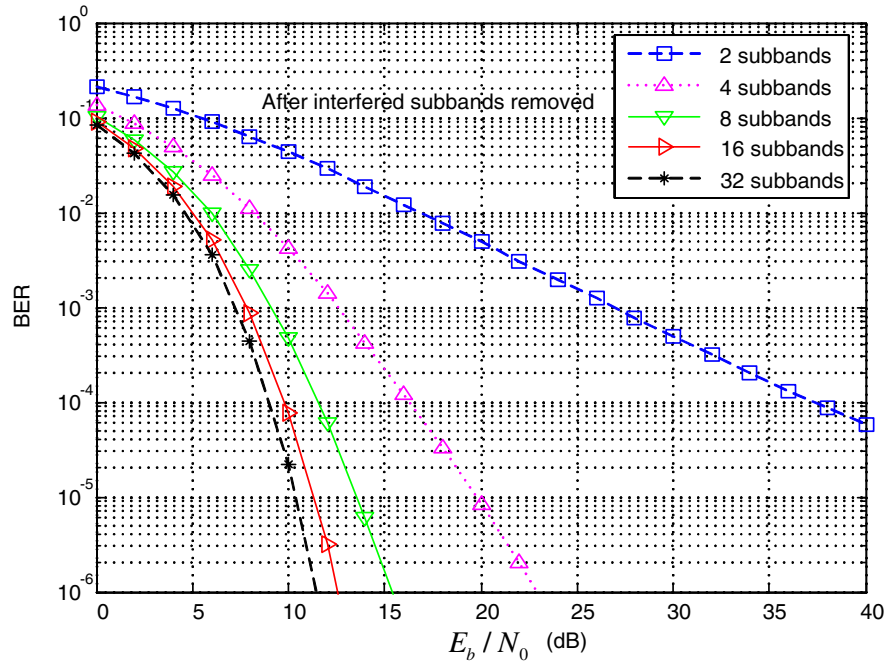

Fig. 7. BER performance after interfered subbands removed.

\section{SYSTEM PERFORMANCE}

Once the system INR thresholds are obtained, the interfered subbands with INR over the thresholds can be adaptively cut off to avoid interference. In order to verify the effectiveness of the subband adaptation in a multipath fading channel, the BERs of the systems without interference, with interference, and with interference removed are simulated. These are displayed in Fig.5, Fig.6 and Fig.7, respectively.

Fig. 5 indicates the system BER performance without interference when system transmission subband numbers $M=2$, $4,8,16$ and 32. It is observed that with the increase of $M$, the system BER performance is improved considerably. After $M=16$, a further increase of transmission subbands is not obviously beneficial to the improvement of the BER performance, but is helpful for the interference avoidance.

Under the same conditions, the system BER with one subband interfered is displayed in Fig.6. The INR is $3 \mathrm{~dB}$. It is seen that the impact from the interference results in $5 \mathrm{~dB}$ $-6 \mathrm{~dB}$ degradation at the BER performance $10^{-6}$ compared with the system performance without interference.

After the interfered subband is removed as displayed in Fig.7, the system BERs are improved $0.5 \mathrm{~dB}-5 \mathrm{~dB}$ compared with the system performance with interference when $M=4$, 8,16 and 32 . In this case the performance is very close to that without interference. However, when $M=2$ the system performance becomes worse, since the subband with INR lower than the threshold $\gamma_{0}=4.9 \mathrm{~dB}$ is removed, which agrees with the previous analysis.

\section{CONCLUSIONS}

In this paper we have investigated an adaptive subband selection method to suppress interference for system coexistence, and demonstrated the improvement on system performance. We have also shown that the interference thresholds over multipath fading channels approximate to the interference 
thresholds over Gaussian channels when the number of transmission subbands is sufficient enough. It is demonstrated that the derivation of interference thresholds over multipath fading channels can be replaced by interference thresholds over Gaussian channels after considering a known discrepancy value. This approximating method greatly simplified derivation of the interference thresholds over multipath fading channels. The proposed algorithms can be applied to support the advancement of cognitive radio and future generation communication applications.

\section{REFERENCES}

[1] K. E. Nolan, P. D. Sutton, L. E. Doyle, T. W. Rondeau, B. Le, and C. W. Bostian, "Dynamic Spectrum Access and Coexistence Expericences Involving Two Independently Developed Cognitive Radio Testbeds," in 2nd IEEE International Symposium on New Frontiers in Dynamic Spectrum Access Networks, pp. 270-275, 2007.

[2] J. Lansford, "UWB Coexistence and Cognitive Radio," in Joint UWBST \& IWUWBS.2004 International Workshop on Ultra Wideband Systems, pp. 35-39, 2004.

[3] X. Jing, S. Mau, D. Raychaudhuri, and R. Matyas, "Reactive Cognitive Radio Algorithms for Co-Existence between IEEE802.11b AND 802.16A Networks," in Global Telecommunications Conference, vol. 5, pp. 2465$2469,2005$.

[4] D. Cabric and R. W. Brodersen, "Physical Layer Design Issues Unique to Cognitive Radio Systems," in IEEE 16th International Symposium on Personal, Indoor and Mobile Radio Communications, vol. 2, pp. 759-763, Nov.2005.

[5] B. Jung, Y. Hong, D. Sung, and S. Chung, "Adaptive Sub-band Nulling for OFDM-Based Wireless Communication Systems," in Wireless Communications and Networking Conference, pp. 1491-1495, 2007.

[6] J. Chiang and J. Lansford, "Use of Cognitive Radio Techniques for OFDM Ultra Wideband Coexistence with Wimax," in Texas Wireless Symposium, pp. 91-95, 2005. http://www.utdallas.edu/ cpb021000/shared/pdfs/0000091.pdf.

[7] P. Tu, X. Huang, and E. Dutkiewiz, "Diversity Performance of an Interleaved Spread Spectrum OFDM System over Frequency Selective Multipath Fading Channels," in 7th International Symposium on Communications and Information Technologies 2007, Sydney, pp.184-189, 2007. 